\title{
Building a Semantic Health Data Warehouse in the Context of Clinical Trials: Development and Usability Study
}

Romain Lelong $^{1,2}$, PhD; Lina F Soualmia ${ }^{1,2,3}$, PhD; Julien Grosjean ${ }^{1,3}, \mathrm{PhD}$; Mehdi Taalba ${ }^{1}$, MD; Stéfan J Darmoni ${ }^{1,3}$, $\mathrm{MD}, \mathrm{PhD}$

${ }^{1}$ Department of Biomedical Informatics, Rouen University Hospital, Rouen, France

${ }^{2}$ LITIS EA 4108, TIBS, Normandy University, Rouen, France

${ }^{3}$ LIMICS U1142, Inserm, Sorbonne University, Paris, France

\section{Corresponding Author:}

Romain Lelong, $\mathrm{PhD}$

Department of Biomedical Informatics

Rouen University Hospital

D2IM, Porte 21, Cour Leschevin

Hôpital Charles-Nicolle, 1 Rue de Germont

Rouen, 76000

France

Phone: 33232888898

Email: romain.lelong@gmail.com

\begin{abstract}
Background: The huge amount of clinical, administrative, and demographic data recorded and maintained by hospitals can be consistently aggregated into health data warehouses with a uniform data model. In 2017, Rouen University Hospital (RUH) initiated the design of a semantic health data warehouse enabling both semantic description and retrieval of health information.

Objective: This study aimed to present a proof of concept of this semantic health data warehouse, based on the data of 250,000 patients from RUH, and to assess its ability to assist health professionals in prescreening eligible patients in a clinical trials context.

Methods: The semantic health data warehouse relies on 3 distinct semantic layers: (1) a terminology and ontology portal, (2) a semantic annotator, and (3) a semantic search engine and NoSQL (not only structured query language) layer to enhance data access performances. The system adopts an entity-centered vision that provides generic search capabilities able to express data requirements in terms of the whole set of interconnected conceptual entities that compose health information.

Results: We assessed the ability of the system to assist the search for 95 inclusion and exclusion criteria originating from 5 randomly chosen clinical trials from RUH. The system succeeded in fully automating 39\% (29/74) of the criteria and was efficiently used as a prescreening tool for 73\% (54/74) of them. Furthermore, the targeted sources of information and the search engine-related or data-related limitations that could explain the results for each criterion were also observed.

Conclusions: The entity-centered vision contrasts with the usual patient-centered vision adopted by existing systems. It enables more genericity in the information retrieval process. It also allows to fully exploit the semantic description of health information. Despite their semantic annotation, searching within clinical narratives remained the major challenge of the system. A finer annotation of the clinical texts and the addition of specific functionalities would significantly improve the results. The semantic aspect of the system combined with its generic entity-centered vision enables the processing of a large range of clinical questions. However, an important part of health information remains in clinical narratives, and we are currently investigating novel approaches (deep learning) to enhance the semantic annotation of those unstructured data.
\end{abstract}

(JMIR Med Inform 2019;7(4):e13917) doi: 10.2196/13917

\section{KEYWORDS}

data warehousing; search engine; semantics; clinical trial; patient selection 


\section{Introduction}

\section{Background and Significance}

Hospitals maintain important health data that can be used in various contexts: first and foremost, clinical care and then data reusability, clinical decision support systems [1], clinical research and cohort selection [2], education [3,4], and indicators. However, the exploitation of these data remains difficult for several reasons. First, the data are produced and maintained by different systems and health professionals and are consequently spread over multiple sources and even across multiple establishments. Second, the significant amount of data generated results in problematic management of data both in terms of data storage capabilities and data access performances. Health data can synthetically and legitimately be described as big data. For instance, according to research $[5,6]$, in the United States, the health care system alone reached 150 exabytes $\left(1.5 \times 10^{20}\right.$ bytes $)$ in 2011 and will reach the yottabyte scale $\left(10^{24}\right.$ bytes $)$ in the near future. Moreover, the health data produced are of different nature; some data are natively structured (eg, diagnosis-related group [DRG] codings and laboratory tests results), but an important part of medical information remains in unstructured free-text clinical narratives (CNs; eg, admission notes, history and physical reports, discharge summaries, radiology reports, and pathology reports) [7]. This unstructured information is particularly relevant in the context of cohort selection tasks. However, in the study by Raghavan et al [8], the authors found that not only unstructured data were essential to resolve between $59 \%$ and $77 \%$ of some clinical trials criteria but also that combining the use of structured and unstructured data enabled leverage of patient recruitment. To process unstructured data, the main approaches rely on natural language processing (NLP) methods $[9,10]$. The background knowledge, as represented in terminologies and ontologies (T\&Os; that describe the domain), plays a crucial role in any clinical NLP task [11]. A common approach to information retrieval (IR) in clinical unstructured text outside the basic full-text search comprises partially restructuring the original texts using semantic annotators (eg, MetaMap [12]) that map words or expressions to concepts from domain knowledge databases.

Consistently aggregating all these scattered, big, complex, and diversely structured data is, in fact, the role of health data warehouses (HDWs). An HDW is defined as a grouping of data from diverse sources accessible by a single data management system [13]. This kind of data repository centralizes clinical, demographic, and administrative data within a uniform and consistent data model. Many HDWs have been proposed worldwide. From a holistic point of view, the majority of these solutions provide aggregated data mainly focusing on patient data as a result. Furthermore, they do not necessarily allow the full and independent visualization and retrieval of the different atomic entities conceptually composing the whole scope of clinical information (eg, Stanford Translational Research Integrated Database Environment or STRIDE [14] and Data Warehouse for Translational Research or DW4TR [15]). This is, nevertheless, particularly important in an IR context, as potential clinical questions and inquiries from health professionals are formulated in terms of their vision of the conceptual organization of data that derive from the actual patient management process. The Enterprise Data Trust [16] relies heavily on industrial solutions to cope with the huge amount of data. Many solutions also implement generic frameworks, such as Informatics for Integrating Biology and the Bedside (i2b2) database. This, however, implies concessions to conciliate the original conceptual representation of data with the data model required by the framework (eg, The European Hospital George Pompidou HDW [17]). Furthermore, many standardized controlled vocabularies used to semantically describe health information do not always provide access to concepts in French, and access to the data through these T\&Os is not always provided for the whole set of data notably as far as the unstructured data are concerned (eg, Electronic Medical Record Search Engine or EMERSE [18] and STRIDE [14]).

In this context, in 2017, the Biomedical Informatics and Information Department of Rouen University Hospital (RUH) initiated the conception and development of a semantic HDW (SHDW). The SHDW functionally relies on 3 independent semantic layers: layer 1 -the cross-terminological health T\&O portal (HeTOP) [19] that provides the background knowledge necessary to semantically describe the health data; layer 2-a semantic annotator, the extracting concepts from multiple terminologies (ECMT) [20,21], that enables the annotation of unstructured data; and layer 3, the semantic search engine (SSE) [22-24] and a Web application interface semantic access to health information, ASIS, that enable access and retrieval of health data through different conceptual entities composing health information. A generic entity-attribute-value (EAV) data model and a not only structured query language (NoSQL) layer (layer 0 ) enable data structuring while preserving the original conceptual data model.

This study aimed to present a proof of concept (POC) of this SHDW based on the data of 250,000 patients from RUH and to assess its ability to assist health professionals in prescreening eligible patients in a clinical trial context. Since November 2018, this POC has integrated all the data of 1.8 million patients from RUH.

\section{Related Studies}

Clinical data warehousing manages health data from hospitals and is a well-addressed research field. Few generic frameworks and components exist. i2b2 $[25,26]$ is a data mart used in $>200$ hospitals worldwide. Initiated within the Massachusetts General Hospital in 2004, i2b2 was developed by the Harvard Medical School and is funded by the National Institutes of Health. It enables the integration of clinical and genomic data into an EAV model known as the star schema. i2b2 enables the retrieval of patients' data using graphically built queries and querying of free-texts and coded information. Another example of a distributed solution is the Observational Medical Outcomes Partnership Common Data Model [27]. This EAV model tends to standardize data from HDW at structure and representation levels (ie, terminologies and vocabularies).

In France, a few open-source solutions exist, such as Dr Warehouse [28] - the CN-oriented data warehouse of Necker Children's Hospital, but 2 solutions really stand out from the 
others: the ConSoRe system [29] used in some French Oncology Hospitals and the query engine Biomedical data warehouse of the hospital whose French acronym is eHOP [30,31] that is being deployed in 6 University Hospitals in Western France.

Owing to the specificity of the data and their private and sensitive aspect, HDWs are specific systems that are used locally in Hospital Information Systems (HISs) rather than distributed and ready-to-use solutions, and many specific HDWs have been developed worldwide in addition to the previously cited generic solutions.

The STRIDE (United States) [14] project focuses on a clinical data warehouse supporting clinical and translational research. It was initiated in 2003 at Stanford University when the functionalities of $\mathrm{i} 2 \mathrm{~b} 2$ and CAncer Biomedical Informatics Grid were not considered optimal. An Oracle database and an EAV data model derived from the Health Level 7 Reference Information Model (RIM) standard are used for data storage and representation. Several (mainly English) standardized terminologies are used to represent important biomedical concepts and their relationships (eg, Systematized Nomenclature Of MEDicine-Clinical Terms or SNOMED-CT, RxNorm, 9th revision of the International Classification of Diseases or ICD-9-Clinical Modification, and current procedural terminology). STRIDE provides hierarchical concept-based retrieval as far as structured data are concerned and provides full-text search access to more than 6 million CNs. The system is based on an n-tiered architecture and the querying of the data is distributed along several client applications whose scope targets patient cohort selection, cohort chart review, clinical data extraction, research data management, and specimen data management. The querying is done graphically using drag and drop interface-based components and returns aggregated data as a result without exposing individual patient data.

EMERSE (Michigan, United States) [18] is an electronic health record-oriented system exclusively providing full-text search capabilities into free-text clinical notes.

The Windber Research Institute (United States) developed the DW4TR [15] system to support multiple translational research projects through highly structured medical information represented in 3 dimensions (namely, clinical data, molecular data, and temporal information). Data are collected into an Oracle Relational DataBase Management System (RDBMS) with an EAV data model and are subsequently hosted in an extensible data model that organizes it into a structure of hierarchical modules inherited from especially developed ontologies. It provides 2 graphical querying interfaces designed to provide aggregated data dedicated to data analysis (eg, mean, standard deviation, counts, categorical data, and chronological view).

The Enterprise Data Trust [16] is an industrial HDW initiated in 2005 at the Mayo Clinic (50,000 employees, Rochester, Minnesota, United States). It collects patient care, education, research, and administrative data to support IR, business intelligence, and high-level decision making. The Enterprise Data Trust strongly relies on industrial technologies (eg, InfoSphere Information Server-International Business Machines; iSight and iGuard-Teleran;

BusinessObjects-Systems Applications and Products; and PowerDesigner-Sybase) and enables integration and exploitation of important volumes of data (eg, more than 7 million unique patients, 64 million diagnoses, and 268 million test results). The architecture and functionalities of the Enterprise Data Trust rely on legacy technical components and long-standing governance works on data and metadata management, data modeling, and standardized vocabularies. Those initiatives provide the HDW with a reliable organization of information on patient, genomic, and research data as well as querying capabilities for cohort selection and aggregate retrieval.

In 2008, the European Hospital George Pompidou (Paris, France) initiated an HDW [17] based on the i2b2 framework. It is strongly integrated in the clinical information system (IS) of the hospital that relies on several industrial solutions (eg, OneCall-McKesson; Act management, computerized physician order entry-Medasys; and integration platform-Thales). The core HDW infrastructure relies on an Oracle database for storage (1.2 million patients and 1 million stays) and the i2b2 framework for data representation. Several client applications are connected to the system to provide technical access to the data but mainly use i2b2 client as far as researchers are concerned. The SMart Eye DATabase (SMEYEDAT) [32] is an ophthalmologic-specialized HDW developed at the University Eye Hospital in Munich in Germany. SMEYEDAT is based on a Microsoft structured query language (SQL) database updated daily from the HIS and uses a star-like patient-centered data model for data representation. The QlikView (QlikTech) [33] tool was implemented as an analytic tool to visualize and explore patient data. This interface enables patient selection using criteria and views specific to the domain.

\section{Methods}

Overall, the first prerequisite pertaining to the design of an HDW-based system is the extraction of data from the HIS. This can be achieved in 2 ways: by (1) setting up a data stream from the production environment (or a replicated database) to the HDW data storage component, or (2) using extract-transform-load (ETL) scripts. As far as the SHDW is concerned, ETL scripts are used. The following section describes the targeted sources of data of the HIS of RUH.

\section{Data Sources}

Since 1992, RUH has collected and maintained patient identity (eg, name, date of birth, and gender), clinical (eg, biological test results, medical procedures, visit records, letters, and discharge summaries), administrative, and less frequently, omics data [34]. The data are produced by different subsystems and applications of the IS of RUH. A subsystem called CPage Dossier Patient partially aggregates some of this important data such as laboratory results, DRGs, procedures, and clinical documents. Other data remain in other subsystems that have to be accessed separately. Overall, RUH maintains the data of 1.8 million patients that represent approximately 14.4 million visits, 11.9 million clinical documents (free-texts recorded since 2000), and 107 million single laboratory tests (eg, Sodium and Potassium being considered as 2 distinct tests; recorded since 
2004). Since November 2018, the SHDW POC presented in this study includes the whole set of data. However, this study is based on a randomly chosen subset of data from 207,357 patients, 1.7 million visits, 671,442 clinical documents, and 14.2 million single laboratory tests. ETL scripts are used to incorporate data from the production environment repositories into an Oracle database. Figure 1 summarizes included data according to their specific domain (ie, reference management, administrative record, care, examinations, health economy, planning and coordination, external data, resource management and billing, sharing, and security). Data already included in the semantic health data warehouse (SHDW) are represented by a dark gray opaque background, whereas a light gray background indicates that data are neither included nor planned to be included in the short or medium term. Background partially or totally covered with bricks corresponds to data for which inclusion is in progress or is planned in the short term or medium term.

Figure 1. Functional coverage of the semantic health data warehouse in terms of data according to each domain. SHDW: semantic health data warehouse; CPOE: computerized physician order entry; DCC: French cancer communication file; PMR: personal medical record; DRG: diagnosis-related group; EHR: electronic health record.
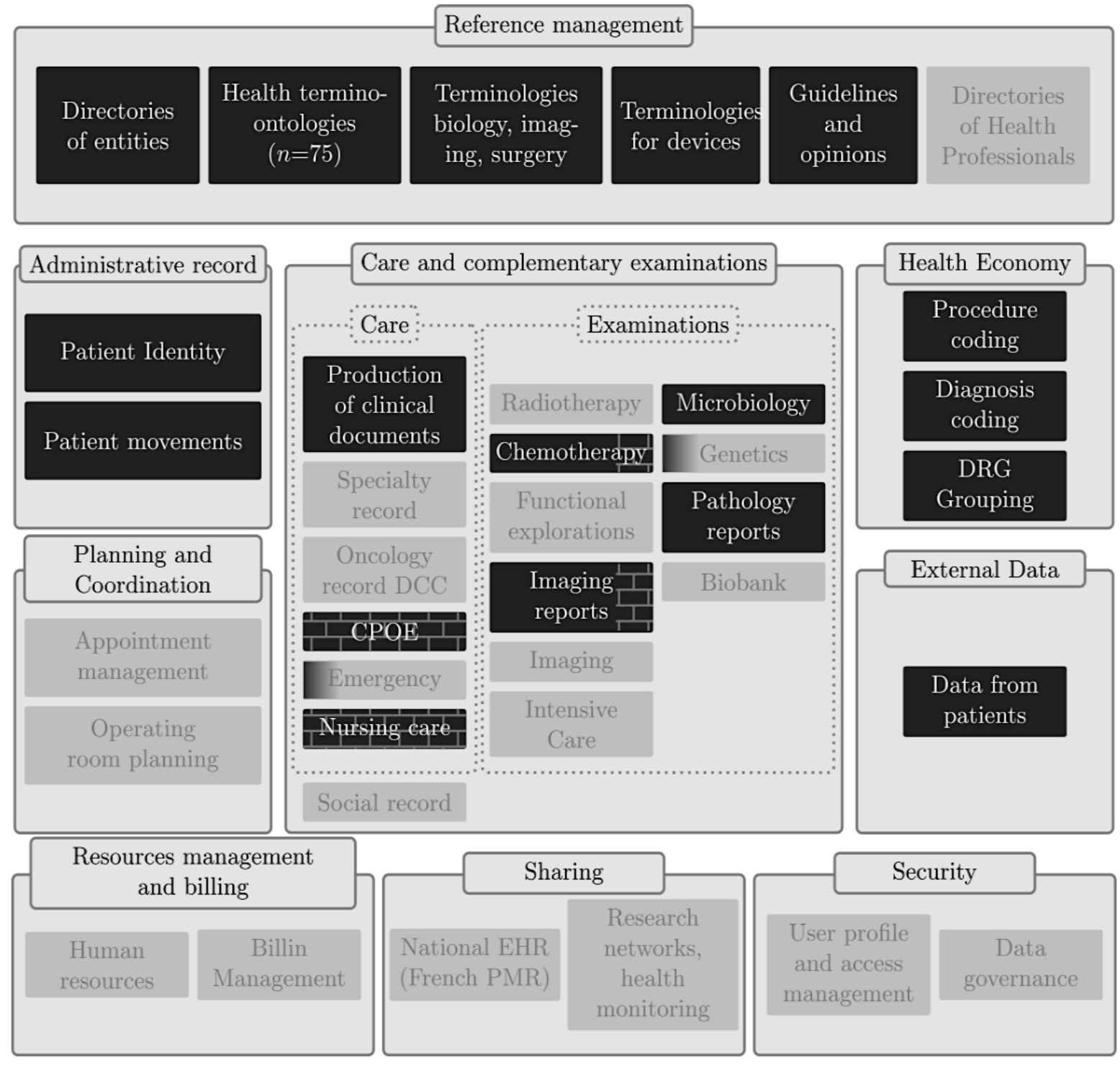

available in the SHDW

not available in the SHDW

ongoing integration

The SHDW currently focuses on clinical data and, more broadly, on health data according to a patient-centered strategy. In addition to the structured patient data, the different data pertaining to multiple admissions and events at RUH are collected (eg, diagnoses, biology, procedures, and movements). The reference-controlled vocabularies (ie, reference management domain) necessary to the understanding of those data are notably widely collected and maintained. In contrast, pure management and administrative data, such as appointment and planning data, billing data, and data governance, are not likely to be included in the short term. All those data are integrated into a modular architecture that is described in the following section. 


\section{Overall Architecture of the Semantic Health Database} Warehouse

Much health information remains in CNs [7]. The 11.9 million clinical documents in French of RUH consequently play a strategic role in the context of the SHDW. Since its creation in 1995, our research team has strongly investigated French IR research domains through T\&Os (and more broadly knowledge organization systems or KOSs), which has led to the development of several search tools mostly dedicated to IR from documentary and bibliographical resources $[22,35]$. However, the complexity of the clinical data and, more broadly, of SHDWs as a whole required the pooling of several of these acquired skills and tools. The SHDW enables the semantic retrieval of health data in French based on several T\&Os and consequently relies on 2 datasets: a domain knowledge database and a health database maintaining clinical and patient data. The functionalities of the SHDW are ensured by the collaboration of 3 distinct layers, where each layer consumes data from the above layers (see Figure 2): the (1) cross-terminological HeTOP [19], (2) semantic annotator ECMT [20,21,36], and (3) SSE [22-24].

Figure 2. Functional architecture of the semantic health data warehouse that provides semantic information retrieval (IR) functionalities form clinical data. The 2 data repositories, knowledge data and health data, respectively, maintain the reference knowledge organization systems and the health data pertaining to the semantic health data warehouse. These data are accessed through a not only structured query language (NoSQL) layer by the 3 distinct components: the cross-terminological health terminology and ontology portal (HeTOP), the semantic annotator extracting concepts from multiple terminologies (ECMT), and the semantic search engine (SSE), each operating on a different range of data. CN: clinical narrative; T\&O: terminology and ontology.

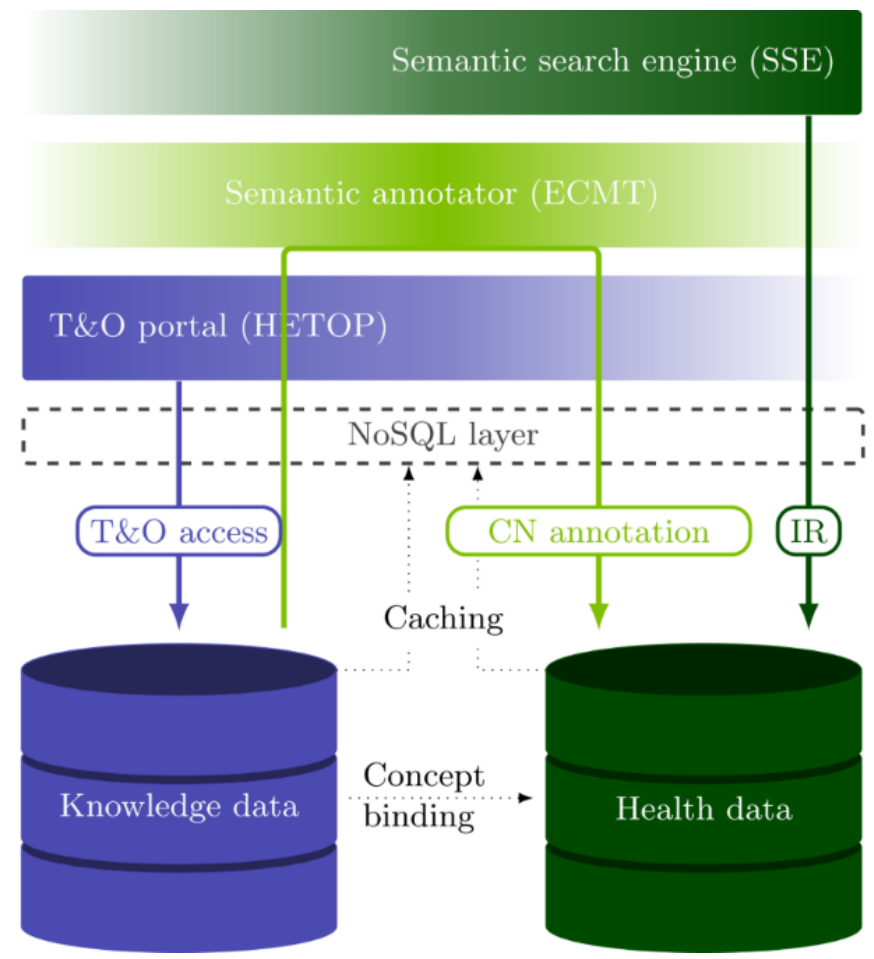

The HeTOP provides access to domain knowledge data. The ECMT matches words and expressions in natural language to domain knowledge concepts included in the HeTOP. In fact, ECMT enables the extraction of semantic information from unstructured data. Its functional scope consequently lies between domain knowledge and clinical data. Together, the 2 components HeTOP and ECMT serve as a base for the semantic description of the clinical information in a computer-processable form. In contrast, the SSE and the coupled Web application, are dedicated to IR tasks on health data by using this extracted semantic description.

Considering the amount of data, access to health data and domain knowledge data is made through an NoSQL layer [22] based on the Infinispan solution, an in-memory data grid (IMDG), on a server with 192 cores and $1 \mathrm{~TB}$ (ie, $10^{12}$ bytes) of random access memory (RAM) allowing vertical scaling.

Each of these layers is functionally and technically detailed below.

\section{Semantic Representation}

This section describes data and the methods for data storage and modeling and presents ECMT that enables the link between knowledge data and actual clinical data.

\section{Domain Knowledge Data}

The HeTOP provides cross-lingual access to concepts originating from 75 T\&Os. A set of 2,639,620 concepts and $10,735,905$ terms are available mainly in English and French. However, 32 languages are available overall. Some of the T\&Os have been partially or totally translated into French (eg, SNOMED 3.5-52.3\%; Medical Subject Heading descriptors-100\%; National Cancer Institute Thesaurus-53.35\%; Online Mendelian Inheritance in Man-79.67\%; Human Phenotype Ontology-72.19\%; and RadLex-22.1\%). More broadly, 50\% of the 2.64 million concepts accessible through the HeTOP are provided in French, and $19.1 \%$ of the 10.74 million terms have a French translation. T\&Os from the HeTOP come with their original sets of 
hierarchies and semantic relationships but also with additional cross-terminological exact, broader to narrower, and narrower to broader mappings performed manually or supervised by our health professionals at RUH.

As a primary use, the different concepts are bound to the different clinical entities (eg, procedure and DRG codings, and $\mathrm{CN}$ annotations), thus allowing a semantic description of the clinical information to be obtained. This allows both refining and broadening of the IR tasks by exploiting the underlying semantic network formed by the concepts (ie, by controlling the granularity and the depth with which this semantic network should be browsed in search processes).

\section{Health Data Model}

Health data are stored in a PostgreSQL [37] relational database. A generic and very adaptable physical EAV data model [34,38] is used to integrate the data. This model structures the information in terms of objects, attributes, and relationships and thus defines an underlying entity-association modeling of the data. It enables the preservation of any original conceptual organization of the information without altering the physical data model and consequently maintains the desired vision of the data at conceptual level. A partial and simplified representation including the main entities and a limited number of relationships and attributes of the conceptual data model used for this study is shown in Figure 3. This model is used on a daily basis to satisfy the information needs of the different health professionals of RUH.

Figure 3. Partial Conceptual Model of the semantic health data warehouse represented as a directed and attributed graph. Entities corresponding to elements from terminologies and ontologies are represented with dashed outlines. DRG: diagnosis-related group; PIN: personal identification number.

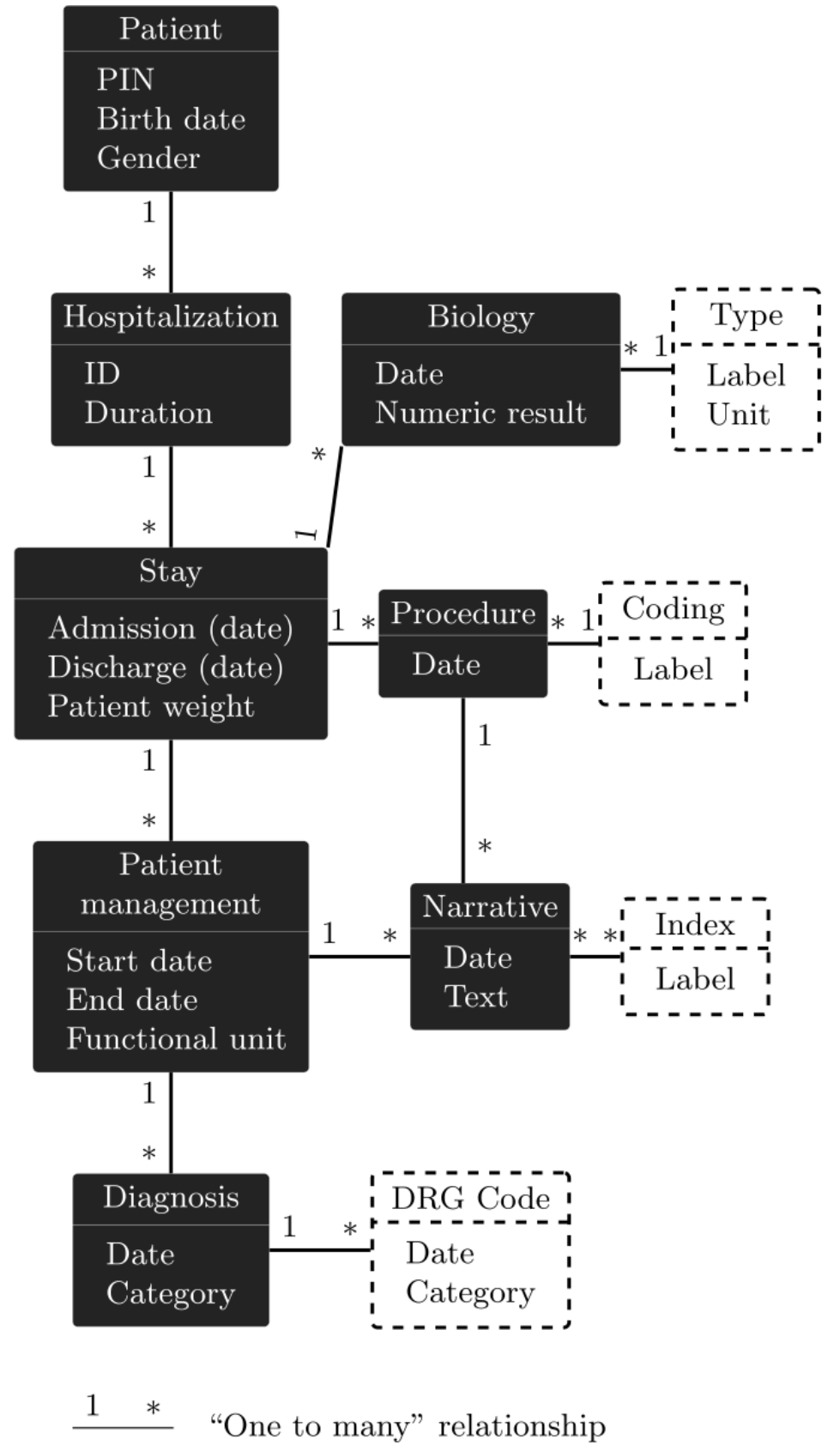




\section{Semantic Annotator}

The semantic annotator ECMT [20,21,36] matches the natural language words and expressions to the domain knowledge concepts included in the HeTOP. Technically, the ECMT relies on the bag-of-word method for concept matching but also provides pattern-matching functionalities, in particular, to deal with negation and contextual information such as numerical values in CNs. Functionally, the ECMT is used in query building processes to match user inputs to accurate sets of concepts but plays a major role in $\mathrm{CN}$ indexing.

\section{Semantic Retrieval}

Access to the data is allowed by a NoSQL layer before processing by the SSE.

\section{Not Only Structured Query Language Layer}

Owing to the considerable amount of health data that need to be retrieved and the well-known limitations of the RDBMSs in terms of scalability, a NoSQL layer was designed to interface access to all the data and improve data access performances. This layer is based on the IMDG Infinispan [39,40]. It is a Java NoSQL solution that uses key-value hash tables as storage structure, which allows efficient recovery of unitary data via the associated keys. Moreover, the hash tables are stored in memory and not on disk, which leverages access times.

The NoSQL layer was conceived in a generic way to mirror the EAV data model used to structure health data (ie, Java object used as values in hash tables mimics the objects and relationships of the relational databases). This generic NoSQL layer consequently preserves the conceptual data model of health and knowledge data implicitly drawn by the EAV data model. A more detailed description of this layer and an overview of performance gain compared with relational RDBMSs are presented in the study by Lelong et al [22].

\section{Semantic Search Engine}

The main purpose of the SSE is to deal with the multiplicity and the diversity of conceptual entities inherent to clinical and patient data (eg, patients, stays, CNs, diagnosis, and biological tests). Overall, the entire set of data originating from the SHDW can be seen as a comprehensive oriented attributed graph that can be queried by the SSE. The SSE was designed to concentrate on semantic retrieval by allowing navigation through the semantic networks, not only included in the T\&Os but also those representing clinical data conceptual entities. From a more clinically coherent point of view, the data of the SHDW can be organized in 4 levels: (1) patient level corresponding to patient identity information, (2) hospital level defining the sources of information (this level is currently not implemented as all the data originate from RUH), (3) visit level that defines much organizational and administrative information about the health care process, and (4) health level enabling group medical procedures and biological tests [24]. As an HDW can be used in various contexts (eg, health care, health research, and secondary use of health data), access and search capabilities of the full scope of those types of information must be provided. Technically, the SSE is a Boolean and entity-oriented search engine. It enables the retrieval and display of data at any of the previous clinical levels. As mentioned in section above, the NoSQL key-value store used to interface data does not provide proper querying solutions. The SSE consequently relies on a specific query language based on formal grammar. It enables the expression of queries targeting any of the different conceptual entities selected through constraints focusing on attribute values and other linked entities [24]. The SSE is used through a Web application that enables the querying of clinical data using forms and string-based queries. This application is described below.

\section{The Semantic Access to a Health Information Web Application: ASIS}

The SSE provides a powerful means to select data using textual logical queries. To bypass the complexity of the query language syntax, we designed a user-friendly Web application known as ASIS. It enables the retrieval of clinical data by means of a form that generates an SSE-processable logic-based query. The clinical data selection process is divided into 4 numbered steps clearly identifiable on the graphical interface. Step 1 comprises building a set of constraints related to any desired entity of interest as patient, diagnosis (DRG), biological tests, stays, procedures, records (CNs), drugs, and medical devices (see Figure 4). Constraints are built via (1) the choice of the entity of interest; (2) the choice of the targeted metadata of this entity as date of birth (patient), gender (patient), type of biological test (biological test), date (eg, procedures, biological tests, and stays), and coding (eg, diagnosis, records, and procedures); and finally, (3) the entry of the inputs corresponding to the chosen entity and metadata as male/female for the gender metadata of a patient constraint and the desired numeric value for the biological test constraint. To facilitate the reading of the interface, each type of entity is represented using a specific color (eg, green for patient, red for diagnoses, green-cyan for biology, and blue for stays). As the SHDW was conceived to focus on semantics, many metadata inputs concentrate on selecting T\&Os and concepts by the user from fields autocompleted to facilitate the selection. For instance, constraints 2 and 3 enable retrieval of CNs indexed with the different concepts referring to type 1 and 2 diabetes (Figure 4). Step 2 comprises aggregating the constraints defined in step 1 into a Boolean query. In this form, constraints are represented as colored buttons showing their IDs, a short description of them, and the numbers of results of the subqueries corresponding to them. A click on a constraint button enables the visualization of the partial results corresponding to the constraints. The step 2 subform editable area enables the composition of the query using parentheses, Boolean operators (ie, AND, OR, and NOT), and the defined constraints that can be selected using an autocompletion feature. Nevertheless, the step 1 subform enables the predefinition of a basic Boolean query skull that is on-the-fly reported in step 2 and can be later manually modified or left untouched in step 2. Step 3 comprises choosing the desired output entity type classified according to the 3 clinical information levels: patient, visit (stay), and health levels. The choice of an entity type generates a button similar to constraint buttons in step 4 . 
Figure 4. The interface of the semantic access to health information, ASIS, Web application, and its 4 steps: (1) definition of constraints, (2) composition of a Boolean query from atomic constraint defined in step 1, (3) selection of the desired output entity according to its clinical coherent level, and (4) visualization of the results.

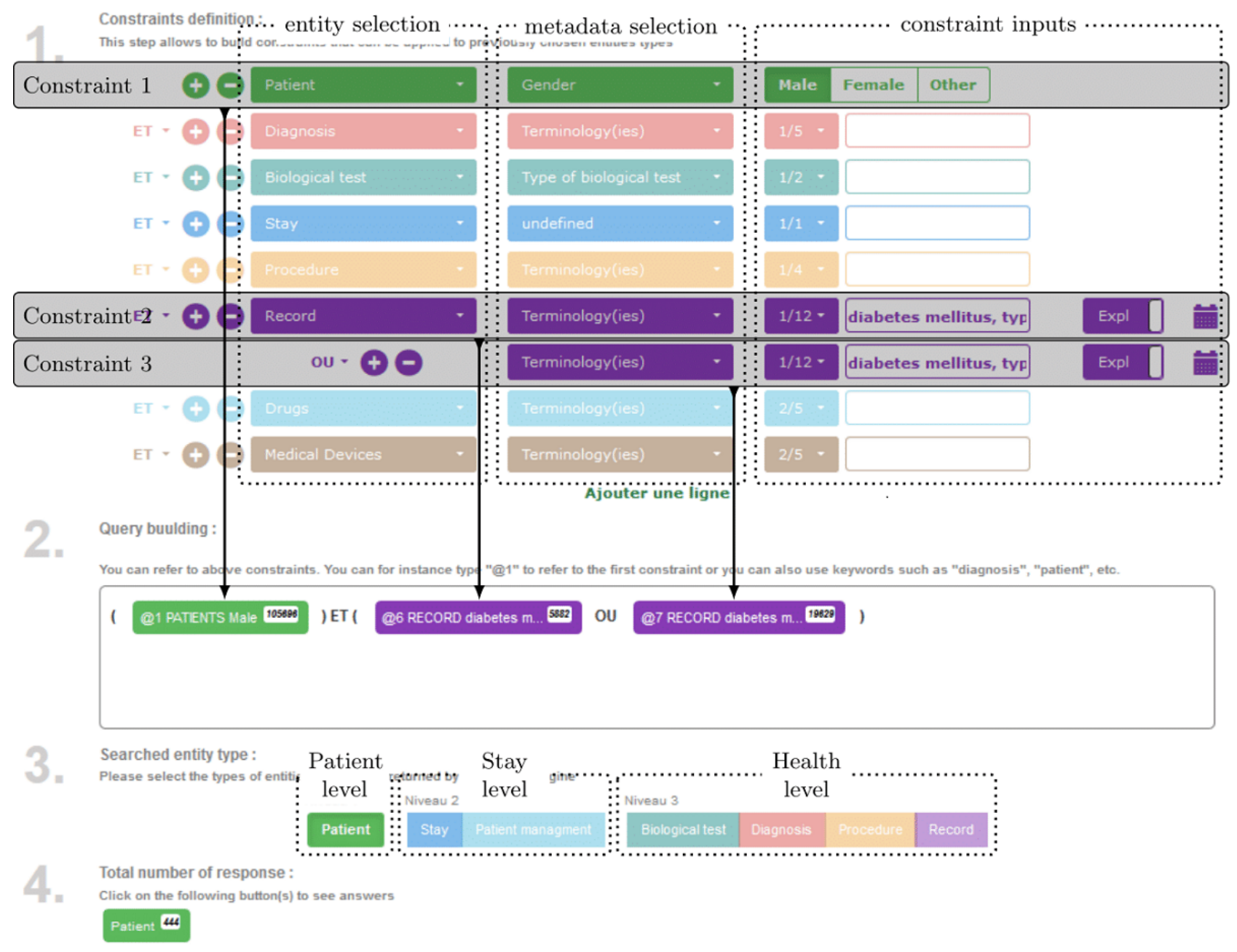

\section{Evaluation Methodology}

A total of 5 clinical trials originating from the RUH, covering a total of 95 criteria (36 inclusions/59 exclusions), were randomly selected without previous information on the content of those clinical trials. The selection was done from a pool of 57 clinical trials initiated between 2005 and 2018 and that were either still recruiting or already completed. The 5 selected clinical trials were initiated in 2012, 2014, 2015, 2016, and 2018 with various medical objectives. Of them, 3 were still in the recruitment phase. The ability of the system to automate patient prescreening was then assessed on each of those criteria, taken independently from both the originating clinical trial and the overall context of the clinical trials. For each criterion, a search strategy was designed. Each of them required the collaboration of a medical doctor (to clinically interpret the criteria and identify the different sources of information to target) and a computer engineer to master the ASIS tool querying process. The search for a single criterion can be done through multiple search directives (ie, ASIS constraints) targeting different sources of information (ie, entities). Those search directives are then aggregated into a single search strategy (ie, a global query) by combining the different search directives using Boolean operations and relational links between the entities corresponding to each search directive. The different constraints that could reduce the accuracy of each search directive were also investigated. In this study, 3 characteristics are finally considered and linked to each other to more precisely identify the different capabilities and limitations of the system: (1) the global support level of the criteria by the system, (2) the targeted source of information, and (3) the obstacle and barriers that tend to lower the effectiveness of the search.

Each of the criteria was, therefore, classified into 6 levels of global support by the system.

\section{Fully Supported Level}

This represents the criteria that can be fully automated by the system with a search strategy that retrieves all and only the resources (without false positives or false negatives) that fulfill the exact requirements of the criteria, for example, 18-year-old patients and patients with a neutrophil level below $1700 / \mathrm{mm}^{3}$.

\section{Accurately Supported Level}

This represents the criteria that are based on consistently recorded data in the IS and on reliable search. The result may, however, possibly include some irrelevant resources or miss relevant ones depending on the choices made in the elaboration process of the search strategy, mainly about the choice of 
concepts to search and the exploitation of their semantic networks, for example, patients with hepatitis $B$ or active hepatitis $C$ and patient with acute kidney failure. In absolute terms, a relevant resource may also be ignored if the data have not been entered in a standard way in the IS.

\section{Broadly Supported Level}

This represents the criteria for which the search results in a lack of precision (ie, inclusion of irrelevant resources or absence of relevant ones). These criteria can only be reliably answered partially. This implies a broadened search of the core requirement of the criteria and a manual postfiltering of the result and/or supervision by a health professional to decide whether, or not, the retrieved resources effectively fit the criteria, for example, patient with an evolving organic digestive and/or inflammatory pathology and patient with a badly regulated cardiac rhythm disturbance.

\section{Inaccurately Supported Level}

This represents the criteria that cannot be searched precisely enough (both technically and in terms of data) to fulfill the core requirement of the criteria or systematically provide consistent results. For example, criterion pregnant woman or breastfeeding mother cannot be searched correctly as the information used to record pregnancy is only very rarely provided as structured data. Thus, the search for information relating to pregnancy and breastfeeding is, therefore, provided mainly in the reports and is, in addition, not systematically provided. This information is sometime provided in a roundabout way involving an effort of deduction or through hardly analyzable natural language expressions. It is, consequently, a difficult information to retrieve. Nevertheless, this information constitutes the essential part of the medical objective of the criterion. Another type of inaccuracy because of technical limitations of the system can also be observed with criterion patient admitted for a stomach hemorrhage resulting in a favorable evolution without surgery during the hospitalization. Although the first part of the criterion is searchable (ie, stomach hemorrhage), the second part of the criterion is not defined with metrics that can be easily interpreted in terms of a query and, more particularly, with regard to favorable evolution. In addition, extensive temporal functionalities would have been necessary, in particular, to exclude favorable evolution following surgery.

\section{Nonsupported Level}

This represents the criteria for which the system fails to properly select the relevant resources (ie, the medical doctor did not consider any of the first results as relevant to the criterion) and/or a search strategy is hardly feasible. For example, the criterion patient with a regular consumption of licorice or derived substances is not supported because the patients' diet is an information which is essentially absent from the IS. In the rare cases where the information appears in the unstructured data, this information is incomplete, unreliable, and technically difficult to identify/extract. Similarly, none of the attempts to define a research strategy to solve the criterion abdominal pain presenting once a week during the last 3 months associated with 2 of the following criteria [...] yielded consistent results.
This criterion involves temporal considerations that are not currently within the scope of the IR system.

\section{Not Applicable and Instruction Level}

This represents criteria that either does not connect to the medical domain or that corresponds more to instructions than real requirements, for example, patient participating in another clinical trial and contraception will be required during the treatment.

A total of 6 types of source of information were identified: (P) Patient structured data as age and gender, (D) DRG data corresponding to structured diagnosis coded with the 10th revision of the ICD and related health problems, (S) stay data and other organizational structured data as medical units, (B) biological structured data, (N) CN unstructured data as full-text and/or automatic indexing including drug data, and (I) for information that is not within the scope of RUH IS.

Finally, the different obstacles or limitations that lower the effectiveness of the search were recorded for each atomic search directive and were distributed among 6 categories: (o) for search directives that are free of any obstacles, (d) for data obstacles corresponding to inconsistently provided or insufficiently accurate data from the IS, (s) for difficulties to perform an accurate search in $\mathrm{CN}$ or DRG data as complex information search, (t) for technical limitations of the system as chronological querying handling or search for quantitative values in CNs (partially implemented), (c) for subjective and/or generic criteria implying the interpretation or value judgment of a health professional, and (e) when it is necessary to meet the patient to complete the criteria.

The global support levels of criteria observed in this study are first detailed in section Global Support of Criteria. A 2-sided Wilcoxon signed-rank statistical test is used to examine the different levels of support of inclusion versus exclusion criteria. The 3 sets of scores detailed in this methodology section are then matched with each other in Observed Sources of Information and Limitations to objectify and identify the concrete abilities and limitation of the system.

\section{Results}

\section{Global Support of Criteria}

As a primary and holistic result, the support levels of the 36 inclusion and the 59 exclusion criteria from the 5 randomly selected clinical trials of RUH are shown in Table 1. The percentage of criteria for each of these levels was recorded.

According to the methodology used to classify criteria, 3 out of the 6 levels of support, full, accurate, and broad could be considered as contributing to cohort's prescreening. Taken together, the system was consequently able, at least partially, to automate the search for 15 out of $36(15 / 36,42 \%)$ of inclusion criteria versus 39 out of $59(39 / 59,66 \%)$ of exclusion criteria. Among the 5 clinical trials used in this study, the number of exclusion criteria exceeded the number of inclusion criteria by $20 \%$ on average (6 vs 16, 9 vs 13, 5 vs 4,7 vs 13, and 9 vs 13 inclusion vs exclusion criteria, respectively). 
Table 1. Number, percentage, and 95\% confidence interval of the percentage of criteria for each support level and type (inclusion or exclusion).

\begin{tabular}{lllllll}
\hline Support level & $\begin{array}{l}\text { Inclusion criteria } \\
\mathrm{n}(\%)\end{array}$ & $95 \% \mathrm{CI}$ & $\begin{array}{l}\text { Exclusion criteria } \\
\mathrm{n}(\%)\end{array}$ & $95 \% \mathrm{CI}$ & $\begin{array}{l}\text { Total } \\
\mathrm{n}(\%)\end{array}$ & \begin{tabular}{l}
$95 \% \mathrm{CI}$ \\
\hline Full
\end{tabular} \\
Accurate & $3(16.67)$ & $(4.5-28.8)$ & $5(8.47)$ & $(1.4-15.6)$ & $11(11.58)$ & $(5.1-18.0)$ \\
Broad & $6(16.67)$ & $(4.5-28.8)$ & $19(32.20)$ & $(20.3-44.1)$ & $25(26.32)$ & $(17.5-35.2)$ \\
Inaccurate & $4(11.11)$ & $(0.8-21.4)$ & $6(10.17)$ & $(2.5-17.9)$ & $10(10.53)$ & $(4.4-16.7)$ \\
None & $3(8.33)$ & $(0.0-17.4)$ & $7(11.86)$ & $(3.6-20.1)$ & $10(10.53)$ & $(4.4-16.7)$ \\
Not applicable & $14(38.89)$ & $(23.0-54.8)$ & $7(11.86)$ & $(3.6-20.1)$ & $21(22.10)$ & $(13.8-30.4)$ \\
Total & $36(100.00)$ & $-{ }^{\mathrm{a}}$ & $59(100.00)$ & - & $95(100.00)$ & - \\
\hline
\end{tabular}

${ }^{\mathrm{a}}$ Not applicable.

A fairer and more reliable measure was also investigated. Of the 95 criteria, $21(21 / 95,22 \%)$ of this study were actually not applicable (N/A) criteria. This type of criteria is not in the scope of an HDW-based system and should consequently be set aside. Moreover, 14 of these 21 criteria (67\%) were attributed to inclusion criteria. Excluding N/A criteria, the percentages of criteria for which the system was able to contribute increased to $68 \%$ for inclusions (15/22 criteria) and $75 \%$ for exclusions (39/52 criteria). When only considering support levels that did not imply postfiltering (ie, only full and accurate), 9 out of 22 $(9 / 22,41 \%)$ inclusion criteria could be answered compared with 20 out of $52(20 / 52,38 \%)$ exclusion criteria.

A 2-sided Wilcoxon signed-rank statistical test was used to compare the levels of support of inclusion versus exclusion criteria. To perform that test, a mean support score was calculated for each subset of inclusion or exclusion criteria of each clinical trial. The calculation of these means was made by assigning to each support level a score from 0 to 100 . The test was not significant with a homogeneous distribution of the scores, but a trend was observed toward better support of inclusion criteria compared with exclusion criteria for distributions that weighted full criteria twice as much as the others. The mean support score of inclusion criteria was constantly greater than that of exclusion criteria for each clinical trial. The tests resulted in observed statistics $\mathrm{T}=15$ with a $P$ value equal to .06 , which, even if slightly greater than the $5 \%$ significance level, suggested a better support of inclusion criteria.

\section{Observed Sources of Information and Limitations}

The results obtained in Table 1 should, nevertheless, be regarded more qualitatively than quantitatively as regard the $95 \%$ confidence intervals that show widths of $20.37 \%$ on average (15.08\% when inclusion and exclusion criteria are taken together). To achieve that goal, both the targeted sources of information and the observed limitations for each support level of each criterion were investigated.

The support level of the criteria according to the combination of information sources required to search them are displayed in Table 2.

Table 2. Number of criteria of each support level according to the combination of sources necessary to search them. The sources of information are as follows: P: patient data, D: diagnoses-related group data, S: stay data, B: biological data, N: clinical narrative, and I: other information.

\begin{tabular}{|c|c|c|c|c|c|c|c|c|c|c|c|c|c|c|c|}
\hline Support level & $P$ & $S$ & $B$ & $D$ & $N$ & $I$ & $S+N$ & $P+N$ & $D+B$ & $B+N$ & $D+N$ & $N+I$ & $P+S+D$ & $S+D+N$ & $D+N+I$ \\
\hline Full & 4 & 0 & 7 & 0 & 0 & 0 & 0 & 0 & 0 & 0 & 0 & 0 & 0 & 0 & 0 \\
\hline Accurate & 1 & 0 & 0 & 8 & 0 & 0 & 0 & 1 & 3 & 0 & 5 & 0 & 0 & 0 & 0 \\
\hline Broad & 0 & 2 & 2 & 6 & 7 & 0 & 1 & 0 & 0 & 1 & 4 & 0 & 0 & 2 & 0 \\
\hline Inaccurate & 0 & 0 & 0 & 0 & 5 & 0 & 0 & 0 & 0 & 1 & 1 & 0 & 1 & 1 & 1 \\
\hline None & 0 & 0 & 0 & 0 & 2 & 3 & 0 & 0 & 0 & 0 & 3 & 1 & 0 & 1 & 0 \\
\hline Not applicable & 0 & 0 & 0 & 0 & 0 & 21 & 0 & 0 & 0 & 0 & 0 & 0 & 0 & 0 & 0 \\
\hline Total & 5 & 2 & 9 & 14 & 14 & 24 & 1 & 1 & 3 & 2 & 13 & 1 & 1 & 4 & 1 \\
\hline
\end{tabular}

Setting aside N/A criteria, 47 out of the 74 criteria $(47 / 74,64 \%)$ could be answered using a single search directive (ie, by exploiting a single source of information) against 27 criteria (36\%) that required combined search directives. The calculation of the mean scores of level of support of these 2 groups of criteria resulted in scores between accurate and broad. Of single search directives, $23.40 \%$ versus $0 \%$ of combined search directives concerned fully supported criteria.
The different sources of information were not uniformly distributed. Patients (P) and stays (S) structured data were involved in the search for only 7 out of the $95(7 / 95,7 \%)$ and 8 out of the $95(8 / 95,8 \%)$ criteria, respectively. In contrast, the top 2 sources of information, $\mathrm{CNs}(\mathrm{N})$ and diagnoses (D), were involved in the search of $37(37 / 95,39 \%)$ and $36(36 / 95,38 \%)$ of the 95 criteria, respectively. 
The percentages of involvement of sources of information and the percentages of involvement of observed limitations for each support level are presented in Figure 5.

Only continuously provided and fully structured data were used to answer fully supported criteria. The only sources of information used were patient structured data $(\mathrm{P})$ and biological data (B). Fully supported criteria were consequently based on very precise characteristics not subject to errors or ambiguity and relying on numeric or symbolic data such as female or male patient aged 18 to 75 years and patient with glycated hemoglobin $\leq 6.5 \%$ or $\geq 8 \%$.

Accurately supported criteria were mostly searched in DRG data (D). In practice, these criteria either rely on a single source of information (eg, HIV-positive patient and type 2 diabetic subject) or the combination of data consistently provided or properly coded as "known active hepatopathy, [...], transaminase and/or alkaline phosphatase levels twice the normal level of the laboratory" (DRGs and biological data) or men aged 18 to 70 years or women aged 18 to 70 years in menopause (patient data and $\mathrm{CNs}$ ).

From a holistic point of view, we observed that DRGs (D) and $\mathrm{CNs}(\mathrm{N})$ were the 2 major sources of information used. As stated before, both were involved in the search strategy of approximately $38 \%$ of criteria (58\% if taken together). The support of the criteria by the system decreased as the exploitation of CNs (N) took precedence over DRG data (D). In this study, the search within $\mathrm{CNs}(\mathrm{N})$ were performed through both full-text and semantic searching. As far as semantic searching is concerned, the $11,928,168 \mathrm{CNs}(\mathrm{N})$ of RUH have been indexed using ECMT over 55 terminologies available in French from the HeTOP server. These CNs were first collected from the RUH IS in their original format (ie, as Microsoft Word files). The raw text was then extracted from these Word documents and stored in simple text files that were provided as input to the ECMT semantic annotator. After some performance optimizations of this annotator, the indexing of all the $\mathrm{CNs}$ could be completed in slightly less than 24 hours on a machine equipped with 1 TB of RAM and 144 cores. The insertion of the annotations in the database was done separately. Indexes allowing retrieval were also generated. The indexing process resulted in a total of 5,043,731,628 annotations. Some of the most redundant concepts were found clinically irrelevant, and a manual filtering process was applied based on the top 5000 most frequent medical concepts (eg, the 27 million annotations with "university hospital" were considered as irrelevant as the information was present elsewhere in the SHDW). A total of $2,087,784,055$ annotations were retained after the filtering process. This set of semantic annotations served as a basis for the SSE in the semantic retrieval process.

Figure 5. The central gray band gives the percentage of criteria of each support level excluding not applicable criteria. The upper bars show, for each support level, the percentages of involvement of each source of information in the search of criteria. The lower bars show the distribution (in percentage) of the different obstacle categories identified as lowering the effectiveness of the search of criteria. CN: clinical narrative; DRG: diagnosis-related group. Distribution of necessary sources of information

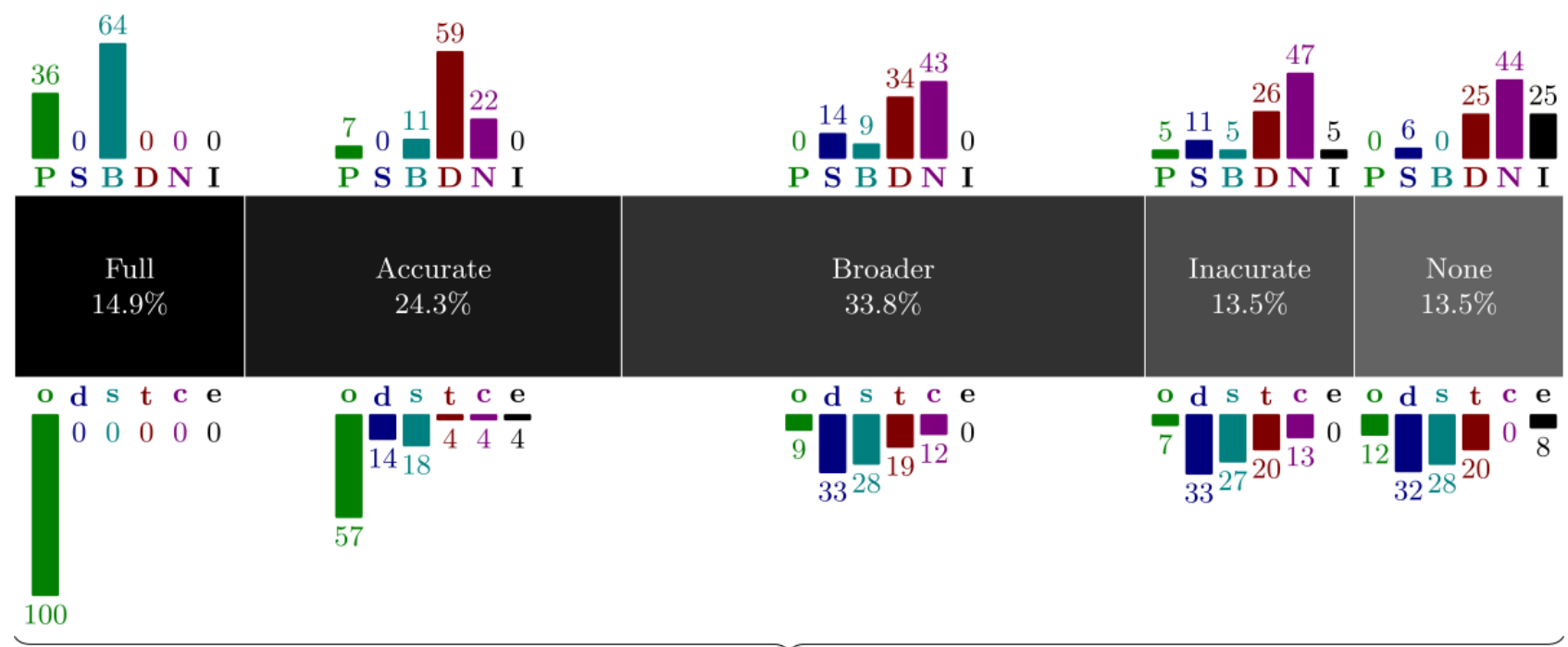

Distribution of observed limitations

Sources of information:

P Patient data

S Stay data

B Biology data

D DRG data

N CN unstructured data

I External information

\author{
Limitations: \\ o No obstacles \\ d Inconsistent data \\ s Complex search \\ t Technical limitations \\ c Subjective or generic criteria \\ e Patient appointment necessary
}


The search accuracy obstacles (s) category represented $20.2 \%$ of all obstacles (32/158), and 84\% (27/32) of these obstacles were attributed to $\mathrm{CN}$ search $(\mathrm{N})$. However, the exploitation of the semantics (ie, synonyms, hierarchical, and semantic relationships) through the automatic indexing of $\mathrm{CNs}(\mathrm{N})$ by the ECMT and the ability of the SSE to combine multiple search directives (using Boolean operators) enabled a broad search support of 25 criteria out of $95(25 / 95,26.3 \%)$. Even when postfiltering was required, the system could be used effectively as a prescreening tool. For instance, the search for the criterion patients with severe heart failure (including New York Heart Association or NYHA Classes III and IV) was done through the search for heart failure in DRG data (D) and the search for NYHA Classes III and IV in CN data (N). Separately, it resulted in 11,880 diagnoses and $3311 \mathrm{CNs}$. The combination of both search directives into a single search enabled the prescreening of only 36 patients.

Data inconsistency (d) was also a major challenge, as 37 obstacles out of the 158 obstacles $(37 / 158,23.4 \%)$ were of that type and found across different sources of information including 10 of those $(10 / 37,27 \%)$ for DRGs (D) and 6 of those (6/37, $16 \%$ ) for stays (S). Many data are sparsely recorded in the IS even outside CNs (eg, weight of the patient as structured data for each stay or S, diet plan in CNs, and hypersensitivity to substances in DRG data or D).

This lack of consistency of information tends to explain the focus on $\mathrm{CNs}(\mathrm{N})$ of inaccurately supported (Inaccurate level) and nonsupported (None level) criteria. In practice, these criteria suffer from the association of concurrent obstacles, often including a data consistency obstacle (d). For instance, both data inconsistency (d) and technical limitations (t) were found for the nonsupported criteria regular consumption of alcohol exceeding $60 \mathrm{~g}$ per day. Information on alcohol consumption was in fact not provided consistently in CNs (N), and technically, it would have required the following: (1) the extraction of a quantitative value from $\mathrm{CNs}$ and (2) the processing of this value as data (partially implemented). As another example, the criterion patient with a creatinine clearance $\leq 50 \mathrm{ml} / \mathrm{mn}$ according to Cockroft formula was inaccurately managed by searching instead for the biological tests of creatinine higher than $100 \mu \mathrm{mol} / \mathrm{L}$. The criterion strongly relies on specific calculation functionalities not provided by the system and is based on sparsely provided data (eg, weight of the patient).

With regard to efficiency, the NoSQL layer used to access the data gave querying performances that were considered extremely satisfactory. On the basis of the data of 250,000 patients, each of the search directives used for this study took less than 2 seconds. As far as the POC integrating the entire patient dataset (1.8 million patients) is concerned, similar performances were observed except for some specific queries targeting and returning huge amounts of biological tests which exceeded 1 $\min$

\section{Discussion}

\section{Principal Findings}

To our knowledge, no formal evaluation of the criteria for clinical trial inclusion and exclusion has been performed using an SHDW. The system based on an SHDW presented in this study could be successfully used to fully automate 29 criteria out of the 74 non-N/A criteria (29/74, 39\%). Moreover, with a limited postfiltering process, it could be efficiently used as a prescreening tool for 54 of those $(54 / 74,73 \%)$.

A trend was observed toward better support of inclusion criteria compared with exclusion criteria for distributions of scores that weighted full criteria twice as much as the others. However, with homogeneous distributions of scores, no conclusion could be made. A lower support of inclusion requirements tends to affect the ability of the system more to assist prescreening tasks. Manual exclusion of patients is usually a lighter task than manual inclusion (especially if the exclusion is made from a small set of patients who already meet the inclusion criteria). Furthermore, clinical trials usually rely on fewer inclusion than exclusion criteria (36 inclusion vs 59 exclusion criteria in this study), which often makes the inclusion requirements more critical prerequisites. Inclusion criteria are indeed used to target specific medical characteristics essential to clinical trials, whereas some exclusion criteria tend to be more generic. For example, one of the clinical trial used in this study included the specific inclusion criteria type 2 diabetic subject and subject with a weight mass index (weight/height ${ }^{2}$ ) greater than 27 and, in contrast, more generic inclusion criteria such as patients with a severe medical or surgical history, in particular, endocrine history or patients treated with drugs interfering with the renin-angiotensin-aldosterone system.

There are still many criteria (ie, 20/74 non-N/A criteria, 27\%) that cannot be searched or can only be partially searched by the system. Several mishandled sources of information along with specific limitations of the system are apt to explain these results. DRG and CN data remain an important source of information for nonsupported or inaccurately supported criteria. Consistent and systematic recording of necessary information in the IS not always performed. Furthermore, this information often resides within the unstructured $\mathrm{CNs}$ form, which is often difficult to extract.

Technically, our system relies only on free solutions. It accesses the data through an IMDG NoSQL layer that offers very satisfactory performances with the data of 250,000 patients. Since November 2018, all the data of the 1.8 million patients from RUH have been integrated into the POC with relatively constant performance (ie, most of the queries tested in this paper are still under the 5-second threshold considered acceptable by health professionals).

\section{Comparison to Prior Work}

The general philosophy of the system relies on a generic representation of clinical information. It enables the independent search and visualization of each conceptual entity (eg, patient, biology, diagnoses, and CNs) that composes the entire health information of the SHDW. Clinical information originating 
from HDWs inherits from a complex data structure. This data structure is very different from documentary and bibliographic IR context, which has been studied in the last 2 decades [22,35] and involves a limited number of entities and relationships and where data are classically more flatly structured with a limited depth (basically, a resource entity possibly surrounded with several other entities, such as an author or an editor entity). We believe that this entity-oriented vision of the SHDW gives added value to the IR systems dedicated to HDW, compared with existing solutions, such as i2b2, which usually adopt a patient-centered vision and provide the user with aggregated data and lists of patients as a result. Notably, the system allows the search to be conducted in an iterative manner by visualizing the search of each entity before aggregating all of them into a comprehensive and coherent search.

In addition, the underlying powerful query language used by the system makes the querying of entity-based co-occurring events more generic and more intuitive (ie, searching several events occurring in the same stay, hospitalization, and medical units) $[23,24]$. In contrast, that kind of functionality is usually proposed through user-friendly but predefined and specific forms (eg, STRIDE [14] and i2b2 [25,26]).

One of the fundamental aspects of SHDW is the semantic description of the health information. This was achieved with the help of many health T\&Os provided by the HeTOP. The ECMT semantic annotator notably enabled to automatically annotate the 11.9 million CNs, and thus, provided a semantic access to these $\mathrm{CNs}$ despite the difficulties to access unstructured information contained within CNs. A bunch of semantic annotators have been proposed for English texts. Recently, Névéol et al [41] performed a literature review on NLP tools in health in languages other than English. In this study, French was the most studied language, followed by German and Chinese. Nevertheless, most of the existing semantic annotators usually extract concepts from the Unified Medical Language System (UMLS) Metathesaurus (eg, MetaMap [12]) [42] or from mainly English T\&Os such as the SNOMED-CT terminology (eg, Text Analysis and Knowledge Extraction System, SNOMED-CT and RxNorm [43], and the National Center for Biomedical Ontology Annotator [44]). French is little represented in the UMLS [45]. The HeTOP includes only 17 KOSs of the 2017 edition of the UMLS. However, the UMLS only manages 11 resources providing concepts in French, and among the 978,233 concept unique identifiers of the UMLS included in the HeTOP, only 143,762 (143,762/978,233, 14.7\%) concepts in French originate from the UMLS. In contrast, the HeTOP provides access in French to 428,854 of them (428,854/978,233, 43.8\%; almost 3 times more than the UMLS).

The vast majority of HDWs are based on RDBMS (eg, i2b2, STRIDE, DW4TR, SMEYEDAT, and Dr Warehouse). In contrast, the system proposed in this study relies on a NoSQL solution that overcomes the limitations of RDBMS as far as the scalability of data is concerned.

\section{Limitations}

From a holistic point of view, the level of support clearly decreased as the $\mathrm{CNs}$ became the predominant source of information. The exploitation of unstructured data $(\mathrm{N})$ is consequently considered as the major challenge for the SHDW in this study. More advanced methods of information extraction from those unstructured data, such as the extraction and exploitation of quantitative values from $\mathrm{CNs}$ (which is only partially implemented in our system) or the on-the-fly computation of relevant measures (eg, body mass index), could drastically improve the capabilities of the system.

Furthermore, despite the growing interest in statistical machine learning methods, rule-based NLP methods remain predominant as far as clinical information extraction is concerned mainly because of their potential of interoperability and interpretability $[9,10]$. Nevertheless, since 2018, our team has engaged new research on the semantic annotator ECMT to investigate the development of a hybrid approach between bag-of-words algorithm and word embeddings.

Temporal and chronological aspects are a topic of interest of many IR systems and particularly relevant to IR in clinical data [15]. For instance, DW4TR fully integrates temporal aspects to data modeling by the mean of 3 types of temporal information (eg, static, events, and intervals). Together with clinical and biological data, temporal information is 1 of the 3 dimensions of the 3-dimensional representation of health data in DW4TR. Temporal querying (ie, querying data occurring at a definite moment in time) can be achieved by the underlying search engine of the SHDW and its associated specific query language, but the ASIS Web interface still needs to be enhanced to provide specific forms able to generate the entire set of proper string-based queries. In contrast, the querying of chronologically co-occurring events (ie, searching events occurring before, after, at the same time, or within a definite time frame compared with another) is not well supported. Our department is currently discussing generic technical upgrades of the SSE that will enable us to overcome those limitations and also offer powerful functionalities beyond the scope of time handling.

One of the major drawbacks of NoSQL layer used for data access (ie, Infinispan), and more generally, of many in-memory key-value stores, is that no comprehensive query language is provided as opposed to the SQL for RDBMS. Complex querying capabilities must be fully implemented from the basic application programing interface (ie, obtaining and removing a value of a specific key) proposed by this kind of solution. In particular, in this study, neither join nor reverted index functionality is natively fully provided by Infinispan and requires, respectively, the maintenance of custom maps and the use of Lucene [46] tools to enable the search from concrete values (ie, text and numerical and data values).

An optimized version of the SHDW is, nevertheless, currently in progress.

\section{Conclusions}

An HDW is defined as a grouping of data from diverse sources accessible by a single data management system [13] that centralizes clinical, demographic, and administrative data within a uniform and consistent data model. In this study, a POC of an SHDW based on the data of 250,000 patients from RUH is presented along with a graphical interface semantic access to health information. The system provides semantic IR capabilities 
and relies on 3 distinct semantic layers. The system was evaluated for its ability to support prescreening of eligible patients in 5 randomly selected clinical trials from RUH. The system showed encouraging results in accurately automating the search of the criteria and good results when used as a prescreening tool. However, this study underlines some limitations of the system especially in relation to information extraction from unstructured $\mathrm{CNs}$, which is still an essential source of information. Since November 2018, all the data of 1.8 million patients from RUH have been included in the POC, and an optimized version is in progress since July 2019.

\section{Acknowledgments}

The authors are grateful to Nikki Sabourin-Gibbs, RUH, for help in editing the paper.

\section{Conflicts of Interest}

None declared.

\section{References}

1. O'Connor PJ, Sperl-Hillen JM, Rush WA, Johnson PE, Amundson GH, Asche SE, et al. Impact of electronic health record clinical decision support on diabetes care: a randomized trial. Ann Fam Med 2011;9(1):12-21 [FREE Full text] [doi: 10.1370/afm.1196] [Medline: 21242556]

2. Shivade C, Raghavan P, Fosler-Lussier E, Embi PJ, Elhadad N, Johnson SB, et al. A review of approaches to identifying patient phenotype cohorts using electronic health records. J Am Med Inform Assoc 2014;21(2):221-230 [FREE Full text] [doi: 10.1136/amiajnl-2013-001935] [Medline: 24201027]

3. Krasowski M, Schriever A, Mathur G, Blau J, Stauffer S, Ford B. Use of a data warehouse at an academic medical center for clinical pathology quality improvement, education, and research. J Pathol Inform 2015;6:45 [FREE Full text] [doi: 10.4103/2153-3539.161615] [Medline: 26284156]

4. VanLangen K, Wellman G. Trends in electronic health record usage among US colleges of pharmacy. Curr Pharm Teach Learn 2018 May;10(5):566-570. [doi: 10.1016/j.cptl.2018.01.010] [Medline: 29986815]

5. Cottle M, Hoover W, Kanwal S, Kohn M, Strome T, Treister NW. Transforming Health Care Through Big Data. 2013. Transforming Health Care Through Big Data URL: http://c4fd63cb482ce6861463-bc6183f1c18e748a49b87a25911a0555. r93.cf2.rackcdn.com/iHT2_BigData_2013.pdf [accessed 2019-10-02]

6. Raghupathi W, Raghupathi V. Big data analytics in healthcare: promise and potential. Health Inf Sci Syst 2014;2:3 [FREE Full text] [doi: 10.1186/2047-2501-2-3] [Medline: 25825667]

7. Petro J. KevinMD. 2011 Sep 1. Natural Language Processing in Electronic Health Records URL: https://www.kevinmd.com/ blog/2011/09/natural-language-processing-electronic-health-records.html, [accessed 2019-10-02]

8. Raghavan P, Chen JL, Fosler-Lussier E, Lai AM. How essential are unstructured clinical narratives and information fusion to clinical trial recruitment? AMIA Jt Summits Transl Sci Proc 2014;2014:218-223 [FREE Full text] [Medline: 25717416]

9. Wang Y, Wang L, Rastegar-Mojarad M, Moon S, Shen F, Afzal N, et al. Clinical information extraction applications: a literature review. J Biomed Inform 2018 Jan;77:34-49 [FREE Full text] [doi: 10.1016/j.jbi.2017.11.011] [Medline: 29162496]

10. Kreimeyer K, Foster M, Pandey A, Arya N, Halford G, Jones SF, et al. Natural language processing systems for capturing and standardizing unstructured clinical information: A systematic review. J Biomed Inform 2017 Sep;73:14-29 [FREE Full text] [doi: 10.1016/j.jbi.2017.07.012] [Medline: 28729030]

11. Doan S, Conway M, Phuong TM, Ohno-Machado L. Natural language processing in biomedicine: a unified system architecture overview. Methods Mol Biol 2014;1168:275-294. [doi: 10.1007/978-1-4939-0847-9 16] [Medline: 24870142]

12. Aronson AR, Lang F. An overview of MetaMap: historical perspective and recent advances. J Am Med Inform Assoc 2010;17(3):229-236 [FREE Full text] [doi: 10.1136/jamia.2009.002733] [Medline: 20442139]

13. International Organization for Standardization. 2010. Health Informatics — Deployment of a Clinical Data Warehouse URL: https://www.iso.org/standard/45582.html [accessed 2019-10-02]

14. Lowe HJ, Ferris TA, Hernandez PM, Weber SC. STRIDE--An integrated standards-based translational research informatics platform. AMIA Annu Symp Proc 2009 Nov 14;2009:391-395 [FREE Full text] [Medline: 20351886]

15. Hu H, Correll M, Kvecher L, Osmond M, Clark J, Bekhash A, et al. DW4TR: A data warehouse for translational research. J Biomed Inform 2011 Dec;44(6):1004-1019 [FREE Full text] [doi: 10.1016/j.jbi.2011.08.003] [Medline: 21872681]

16. Chute CG, Beck SA, Fisk TB, Mohr DN. The Enterprise Data Trust at Mayo Clinic: a semantically integrated warehouse of biomedical data. J Am Med Inform Assoc 2010;17(2):131-135 [FREE Full text] [doi: 10.1136/jamia.2009.002691] [Medline: 20190054]

17. Zapletal E, Rodon N, Grabar N, Degoulet P. Methodology of integration of a clinical data warehouse with a clinical information system: the HEGP case. Stud Health Technol Inform 2010;160(Pt 1):193-197. [doi: 10.3233/978-1-60750-588-4-193] [Medline: 20841676]

18. Hanauer DA, Mei Q, Law J, Khanna R, Zheng K. Supporting information retrieval from electronic health records: a report of University of Michigan's nine-year experience in developing and using the Electronic Medical Record Search Engine (EMERSE). J Biomed Inform 2015 Jun;55:290-300 [FREE Full text] [doi: 10.1016/j.jbi.2015.05.003] [Medline: 25979153] 
19. Grosjean J, Merabti T, Dahamna B, Kergourlay I, Thirion B, Soualmia LF, et al. Health multi-terminology portal: a semantic added-value for patient safety. Stud Health Technol Inform 2011;166:129-138. [doi: 10.3233/978-1-60750-740-6-129] [Medline: 21685618]

20. Soualmia LF, Cabot C, Dahamna B, Darmoni SJ. SIBM at CLEF e-Health Evaluation Lab 2015. In: Proceedings of the 2015 Conference and Labs of the Evaluation forum. 2015 Presented at: CLEF'15; September 8-11, 2015; Toulouse, France URL: http://ceur-ws.org/Vol-1391/125-CR.pdf

21. Cabot C, Soualmia LF, Dahamna B, Darmoni SJ. SIBM at CLEF eHealth Evaluation Lab 2016: Extracting Concepts in French Medical Texts with ECMT and CIMIND. In: Proceedings of the 2016 Conference and Labs of the Evaluation forum. 2016 Presented at: CLEF'16; September 5-8, 2016; Évora, Portugal URL: http://ceur-ws.org/Vol-1609/16090047.pdf

22. Lelong R, Lina F S, Sakji S, Dahamna B, Darmoni S. NoSQL technology in order to support Semantic Health Search Engine. : April 2018 Presented at: Medical Informatics Europe, MIE 2018; Goteborg, Sweden URL: https://hal. archives-ouvertes.fr/hal-02103574

23. Lelong R, Soualmia L, Dahamna B, Griffon N, Darmoni SJ. Querying EHRs with a semantic and entity-oriented query language. Stud Health Technol Inform 2017;235:121-125. [doi: 10.3233/978-1-61499-753-5-121] [Medline: 28423767]

24. Lelong R, Cabot C, Soualmia LF, Darmoni SJ. Semantic Search Engine to Query into Electronic Health Records with a Multiple-Layer Query Language. In: Proceedings of the Association for Computing Machinery's (ACM) Special Interest Group on Information Retrieval (SIGIR). 2016 Presented at: SIGIR'16; 2016; Pisa, Italy URL: http://medir2016.imag.fr/ data/MEDIR 2016 paper 8.pdf

25. Murphy SN, Mendis ME, Berkowitz DA, Kohane I, Chueh HC. Integration of clinical and genetic data in the i2b2 architecture. AMIA Annu Symp Proc 2006:1040 [FREE Full text] [Medline: 17238659]

26. Murphy SN, Weber G, Mendis M, Gainer V, Chueh HC, Churchill S, et al. Serving the enterprise and beyond with informatics for integrating biology and the bedside (i2b2). J Am Med Inform Assoc 2010;17(2):124-130 [FREE Full text] [doi: 10.1136/jamia.2009.000893] [Medline: 20190053]

27. Hripcsak G, Duke JD, Shah NH, Reich CG, Huser V, Schuemie MJ, et al. Observational Health Data Sciences and Informatics (OHDSI): Opportunities for observational researchers. Stud Health Technol Inform 2015;216:574-578 [FREE Full text] [doi: 10.3233/978-1-61499-564-7-574] [Medline: 26262116]

28. Garcelon N, Neuraz A, Salomon R, Faour H, Benoit V, Delapalme A, et al. A clinician friendly data warehouse oriented toward narrative reports: Dr. Warehouse. J Biomed Inform 2018 Apr;80:52-63 [FREE Full text] [doi:

10.1016/j.jbi.2018.02.019] [Medline: 29501921]

29. Heudel P, Livartowski A, Arveux P, Willm E, Jamain C. [The ConSoRe project supports the implementation of big data in oncology]. Bull Cancer 2016 Nov;103(11):949-950. [doi: 10.1016/j.bulcan.2016.10.001] [Medline: 27816168]

30. Cuggia M, Garcelon N, Campillo-Gimenez B, Bernicot T, Laurent JF, Garin E, et al. Roogle: an information retrieval engine for clinical data warehouse. Stud Health Technol Inform 2011;169:584-588. [doi: 10.3233/978-1-60750-806-9-584] [Medline: 21893816]

31. Delamarre D, Bouzille G, Dalleau K, Courtel D, Cuggia M. Semantic integration of medication data into the EHOP Clinical Data Warehouse. Stud Health Technol Inform 2015;210:702-706. [doi: 10.3233/978-1-61499-512-8-702] [Medline: 25991243]

32. Kortüm KU, Müller M, Kern C, Babenko A, Mayer WJ, Kampik A, et al. Using electronic health records to build an ophthalmologic data warehouse and visualize patients' data. Am J Ophthalmol 2017 Jun;178:84-93. [doi: 10.1016/j.ajo.2017.03.026] [Medline: 28365240]

33. Qlik: Data Analytics for Modern Business Intelligence. QlikView URL: https://www.qlik.com/us/products/qlikview [accessed 2019-02-15] [WebCite Cache ID 76Cws45GA]

34. Cabot C, Lelong R, Grosjean J, Soualmia LF, Darmoni SJ. Retrieving clinical and omic data from electronic health records. Stud Health Technol Inform 2016;221:115. [Medline: 27071889]

35. Darmoni SJ, Thirion B, Leroyt JP, Douyère M, Lacoste B, Godard C, et al. A search tool based on 'encapsulated' MeSH thesaurus to retrieve quality health resources on the internet. Med Inform Internet Med 2001;26(3):165-178. [doi: 10.1080/14639230110064488] [Medline: 11706927 ]

36. Cabot C, Soualmia LF, Grosjean J, Griffon N, Darmoni SJ. Evaluation of the terminology coverage in the french corpus LISSa. Stud Health Technol Inform 2017;235:126-130. [doi: 10.3233/978-1-61499-753-5-126] [Medline: 28423768]

37. PostgreSQL. URL: https://www.postgresql.org/ [accessed 2019-02-15] [WebCite Cache ID 76CxVKdFk]

38. Grosjean J. CISMeF - CHU de Rouen. 2014. Modélisation, réalisation et évaluation d'un portail Multi-terminologique, Multi-discipline, Multi-lingue (3M) dans le cadre de la Plateforme d'Indexation Régionale (PlaIR) URL: http://www. chu-rouen.fr/tibs/wp-content/uploads/th\%C3\%A8se Julien Grosjean final.pdf [accessed 2019-10-02]

39. Infinispan Data Grid Platform. URL: http://infinispan.org/ [accessed 2019-02-15] [WebCite Cache ID 76Cw0We8b]

40. Marchioni F, Surtani M. Infinispan Data Grid Platform. Birmingham, United Kingdom: Packt Publishing; 2012.

41. Névéol A, Dalianis H, Velupillai S, Savova G, Zweigenbaum P. Clinical natural language processing in languages other than English: opportunities and challenges. J Biomed Semantics 2018 Mar 30;9(1):12 [FREE Full text] [doi: 10.1186/s13326-018-0179-8] [Medline: 29602312] 
42. Lindberg DA, Humphreys BL, McCray AT. The unified medical language system. Yearb Med Inform 2018;02(01):41-51. [doi: $10.1055 / \mathrm{s}-0038-1637976$ ]

43. Savova GK, Masanz JJ, Ogren PV, Zheng J, Sohn S, Kipper-Schuler KC, et al. Mayo clinical Text Analysis and Knowledge Extraction System (cTAKES): architecture, component evaluation and applications. J Am Med Inform Assoc 2010;17(5):507-513 [FREE Full text] [doi: 10.1136/jamia.2009.001560] [Medline: 20819853]

44. Noy NF, Shah NH, Whetzel PL, Dai B, Dorf M, Griffith N, et al. BioPortal: ontologies and integrated data resources at the click of a mouse. Nucleic Acids Res 2009 Jul;37(Web Server issue):W170-W173 [FREE Full text] [doi: 10.1093/nar/gkp440] [Medline: 19483092]

45. Névéol A, Grosjean J, Darmoni SJ, Zweigenbaum P. Language Resources for French in the Biomedical Domain. In: Proceedings of the Ninth International Conference on Language Resources and Evaluation. 2014 Presented at: LREC'14; May 26-31, 2014; Reykjavik, Iceland p. 2146-2151 URL: https://www.aclweb.org/anthology/L14-1487/

46. Appache Lucene. URL: https://lucene.apache.org/ [accessed 2019-10-02]

\section{Abbreviations}

CN: clinical narrative

DRG: diagnosis-related group

DW4TR: Data Warehouse for Translational Research

EAV: entity-attribute-value

ECMT: extracting concepts from multiple terminologies

EMERSE: Electronic Medical Record Search Engine

ETL: extract-transform-load

HDW: health data warehouse

HeTOP: health T\&O portal

HIS: Hospital Information System

i2b2: Informatics for Integrating Biology and the Bedside

ICD: International Classification of Diseases

IMDG: in-memory data grid

IR: information retrieval

IS: information system

KOS: knowledge organization system

NLP: natural language processing

NoSQL: not only structured query language

NYHA: New York Heart Association

POC: proof of concept

RAM: random access memory

RDBMS: Relational DataBase Management System

RIM: Reference Information Model

RUH: Rouen University Hospital

SHDW: semantic health data warehouse

SMEYEDAT: SMart Eye DATabase

SNOMED-CT: Systematized Nomenclature Of MEDicine-Clinical Terms

SQL: structured query language

SSE: semantic search engine

STRIDE: Stanford Translational Research Integrated Database Environment

T\&O: terminology and ontology

UMLS: Unified Medical Language System

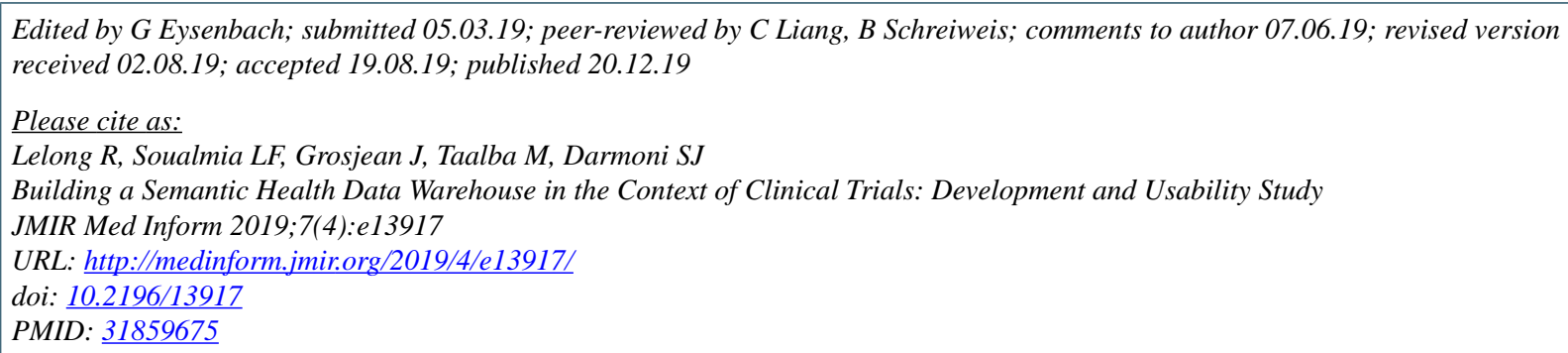


CRomain Lelong, Lina F Soualmia, Julien Grosjean, Mehdi Taalba, Stéfan J Darmoni. Originally published in JMIR Medical Informatics (http://medinform.jmir.org), 20.12.2019. This is an open-access article distributed under the terms of the Creative Commons Attribution License (https://creativecommons.org/licenses/by/4.0/), which permits unrestricted use, distribution, and reproduction in any medium, provided the original work, first published in JMIR Medical Informatics, is properly cited. The complete bibliographic information, a link to the original publication on http://medinform.jmir.org/, as well as this copyright and license information must be included. 